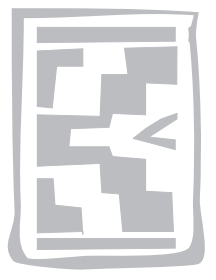

\title{
Effects of differences in virulence of different serovars of Haemophilus paragallinarum on perceived vaccine efficacy
}

R.R. BRAGG

Department of Microbial, Biochemical and Food Biotechnology, University of the Free State

P.O. Box 339, Bloemfontein, 9300 South Africa. E-mail: BraggRR.sci.@mail.uovs.ac.za

\begin{abstract}
BRAGG, R.R. 2005. Effects of differences in virulence of different serovars of Haemophilus paragallinarum on perceived vaccine efficacy. Onderstepoort Journal of Veterinary Research, 72:1-6

The virulence of four South African field isolates of NAD-dependent Haemophilus paragallinarum and two field isolates of NAD-independent $H$. paragallinarum has previously been tested in unvaccinated chickens. In this study, the disease profiles caused by the NAD-dependent isolates of $H$. paragallinarum in vaccinated chickens were studied. It was shown that the clinical signs induced in the vaccinated chickens were substantially less severe than were those in unvaccinated chickens, as was expected. However, due to the high virulence of the serovar C-3 isolates, clinical signs in the vaccinated chickens challenged with this isolate were still detected. These were as severe as those occurring in unvaccinated chickens challenged with serovar B-1 isolates. Although the clinical signs induced in unvaccinated birds challenged with serovar A-1 were more severe than those occurring when vaccinated birds were challenged with serovar C-3, the overall disease profiles were similar. Substantial clinical signs were recorded in vaccinated birds challenged with serovar C-3. This could be interpreted as vaccination failure if the disease profile obtained in unvaccinated birds is not considered. It was found that a high level of protection was provided by this vaccine against challenge by serovar C-3. The high virulence of this serovar resulted in the development of clinical signs in vaccinated birds.

These findings could possibly explain the large number of so-called vaccination failures that are reported in South Africa.
\end{abstract}

Keywords: Haemophilus paragallinarum, immunity, infectious coryza, NAD-independent isolates

\section{INTRODUCTION}

Infectious coryza (IC), caused by the bacterium Haemophilus paragallinarum, was first recorded in South Africa in 1968 on a multi-age layer farm (Buys 1982). The first recorded use of a vaccine against H. paragallinarum in South Africa was in 1975 (Buys 1982), which appeared to effectively control the disease. However, in the mid 1980s, increasing vaccination failures and outbreaks of IC were reported in South Africa

Accepted for publication 1 July 2004-Editor
Bragg, Coetzee \& Verschoor (1996) undertook a serological survey of NAD-dependent $H$. paragallinarum isolates collected over a 30-year period, using the haemagglutination inhibition test first described by Kume, Sawata, Nakase \& Matsumoto (1983) and modified by Blackall, Eaves \& Rogers (1990a). They demonstrated that there had been a significant change in the incidence of the four different South African serovars over a 30-year period from the early 1970s (before the use of vaccines) to the mid 1990s. They demonstrated that the incidence of the NAD-dependent serovar A-1 and C-2 isolates had decreased, while the incidence of 
serovar C-3 had increased significantly. Serovar C-3 has only been reported in South Africa (Kume et al. 1983; Blackall, Eavers \& Aus 1990b; Bragg et al. 1996) and recently in Zimbabwe (Bragg 2002a). None of the vaccines used at that stage contained serovar C-3 and this prompted Bragg et al. (1996) to suggest that the observed changes in incidence were due to the fact that none of the vaccines used contained serovar C-3 and they suggested that there was a need for a local vaccine.

Bragg $(2002 b, c)$ investigated the virulence of naturally occurring NAD-dependent isolates of $H$. paragallinarum and naturally occurring NAD-independent isolates of $H$. paragallinarum in unvaccinated chickens. In these experiments it was demonstrated that the serovar C-3 isolate of NAD-dependent $H$. paragallinarum was highly virulent. The virulence of serovar C-2 was also found to be very high, although lower than that of the serovar C-3 isolate (Bragg $2002 b)$. The virulence of serovar A-1 was lower than expected (Bragg 2002b), while the low virulence of the South African isolate of serogroup B confirmed the results of Yamaguchi, Blackall, Takigami, Iritani \& Hayashi (1990).

When considering the naturally occurring NADindependent isolates, which were first recorded in South Africa in the early 1990s (Horner, Bishop \& Haw 1992; Mouahid, Bisgaard, Morley, Mutters \& Mannheim 1992; Bragg, Coetzee \& Verschoor 1993; Horner, Bishop, Jarvis \& Coetzee 1995; Bragg, Greyling \& Verschoor 1997), it was demonstrated that the virulence of the serogroup $A$ isolates was similar. However, a marked difference in virulence was recorded between the NAD-dependent serovar C-3 isolate and the NAD-independent isolate. The NAD-independent isolate showed much lower virulence. Taole, Albertyn, Van Heerden \& Bragg (2002) demonstrated that laboratory manipulation of naturally occurring NAD-dependent serovar C-3 isolates with a plasmid from naturally occurring NAD-independent isolates resulted in a lowering of the virulence of the transformed strain. Bragg (2004a) demonstrated that the clinical signs caused in vaccinated and unvaccinated chickens challenged with a NAD-independent strain of $H$. paragallinarum were statistically similar, suggesting possible immune evasion by the NAD-independent variants. This is in direct contradiction to the finding of Jacobs \& Van der Werf (2000) who demonstrated that their vaccine provided protection against the NAD-independent isolates of $H$. paragallinarum. It is important to note that different challenge models were used in the different studies.
Using the challenge model described by Bragg (2002b) in which a numerical score can be given to the clinical signs and a disease profile and mean disease score can be calculated, it would be interesting to investigate the effects that prior vaccination has on the ability of the different serovars to cause disease.

The high virulence of the serovar C-3 isolates found by Bragg (2002b) raises the question of whether the increased incidence of these isolates in the 1990s (Bragg et al. 1996) was due to the use of incorrect strains in the vaccine as proposed by Bragg et al. (1996), or due to the elevated virulence of NADdependent serovar $\mathrm{C}-3$ isolates as demonstrated by Bragg (2002b). The objective of the present investigation was to obtain information pertaining to these questions.

\section{MATERIALS AND METHODS}

\section{Chickens}

A total of 80 unvaccinated commercial layer chickens were obtained from a supplier of point-of-lay chickens. These chickens were obtained at 11 weeks of age before they were vaccinated against IC. They were obtained from a farm which had no previous history of IC and were housed in isolated layer facilities at the University of the Free State until they were used in the experiments.

When the birds were 12 weeks of age, 40 of them were randomly selected and vaccinated with an experimental vaccine that contained isolates of all four South African serovars (A-1, B-1, C-2 and C-3) of $H$. paragallinarum in aluminum hydroxide which had been specifically selected based on ability to agglutinate red blood cells (Bragg, unpublished data 2003). They were each vaccinated subcutaneously with $0.5 \mathrm{ml}$ of the vaccine and subsequently revaccinated at 16 weeks of age with the same experimental vaccine. Both the vaccinated and unvaccinated chickens remained in the facilities until they were 25 weeks of age and were producing eggs.

When 25 weeks of age, the vaccinated and unvaccinated chickens were randomly assigned to one of four groups, each of which consisted of ten vaccinated and ten unvaccinated chickens. They were then placed into layer cages in the isolation facilities of the university in such a way that all ten birds of each group were in a row of five cages. A total of two chickens were placed into each cage. The vaccinated chickens were placed on one side of the 
battery of cages, while the unvaccinated chickens were placed on the opposite side.

\section{Challenge exposure methods}

The chickens were challenged with one of the four different serovars of NAD-dependent $H$. paragallinarum isolates according to the methods described by Bragg (2002b), without modification. Basically, one chicken in the middle cage of the row of five cages was selected and challenged by means of an intra-sinus injection of $0.1 \mathrm{~m} \ell$ of the challenge bacterium. The remaining birds in the group were then infected via an in-contact natural challenge route. The severity of the clinical signs in the birds were recorded and scored for a 20-day period according to the methods described by Bragg (2002b).

The NAD-dependent isolates used for challenge purposes were 49 (A-1), 58 (B-1), 7 (C-2) and 46 $(\mathrm{C}-3)$ and were the same isolates used in the production of the experimental vaccine. All the isolates used have previously been described and serotyped and are the same isolates which were used for the establishment of virulence (Bragg 2002b).

\section{RESULTS}

The results of the scoring of the clinical signs were used to produce a graphic representation of the course of the disease in the vaccinated and unvaccinated chickens. The disease profiles of birds challenged with the NAD-dependent serovar A-1 isolate of $H$. paragallinarum are given in Fig. 1, while those

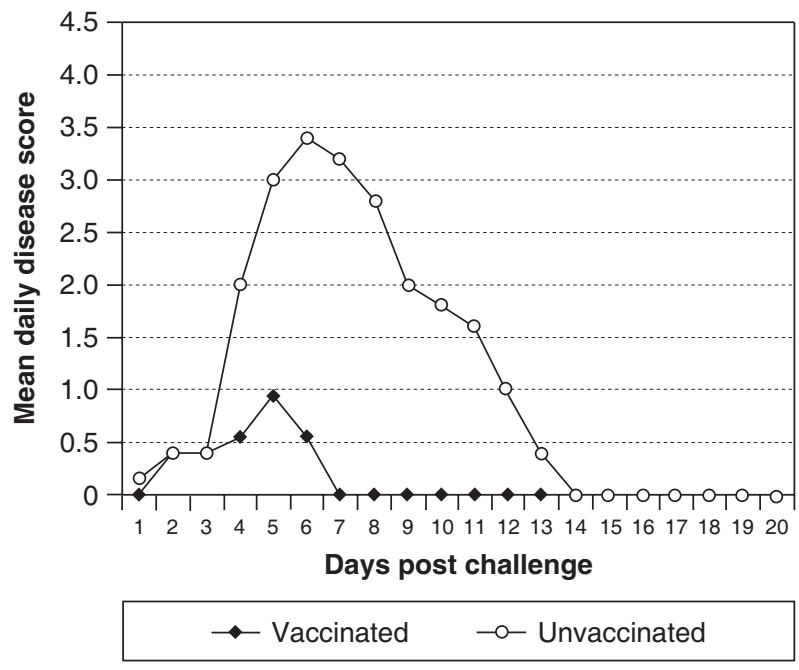

FIG. 1 Disease profiles obtained when vaccinated and unvaccinated chickens were challenged with serovar A-1 of H. paragallinarum of the birds challenged with serovar B-1, C-2 and C3 are reflected in Fig. 2, 3 and 4, respectively. A summary of the mean disease scores, as calculated from the disease profiles in vaccinated and unvaccinated chickens challenged with the different serovars appears in Table 1, as is the maximum disease score for each of the groups of chickens. The duration of the infection, as calculated from Fig.1-4 as the last day on which clinical signs were recorded, is also presented in Table 1.

The percentage protection, as calculated from the mean disease scores of vaccinated chickens versus that of unvaccinated chickens, as well as the result of the statistical analyses of the disease profiles are given in Table 1.

A comparison of the clinical disease profile obtained when vaccinated chickens were challenged with serovar C-3 and the unvaccinated profile of chickens challenged with serovar A-1 can be seen in Fig. 5.

\section{DISCUSSION}

It is evident from the data recorded (Fig. 1-4) that distinct statistically significant (Table 1) differences in the disease profiles for the vaccinated and unvaccinated chickens were obtained.

Similar virulence patterns to those reported by Bragg (2002b) for NAD-dependent strains of $H$. paragallinarum were recorded in the unvaccinated chickens, although the mean disease scores in this experiment were found to be higher for all the isolates.

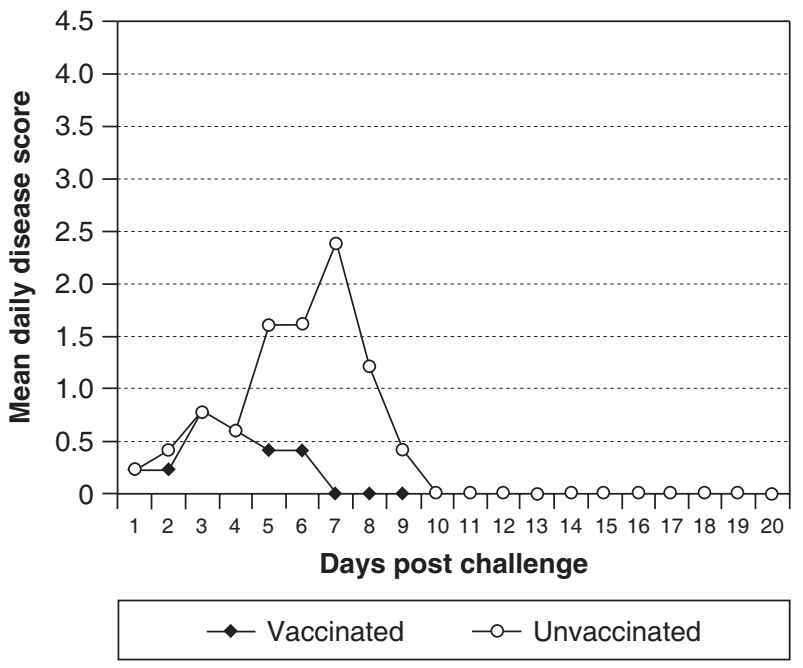

FIG. 2 Disease profiles obtained when vaccinated and unvaccinated chickens were challenged with serovar B-1 of H. paragallinarum 


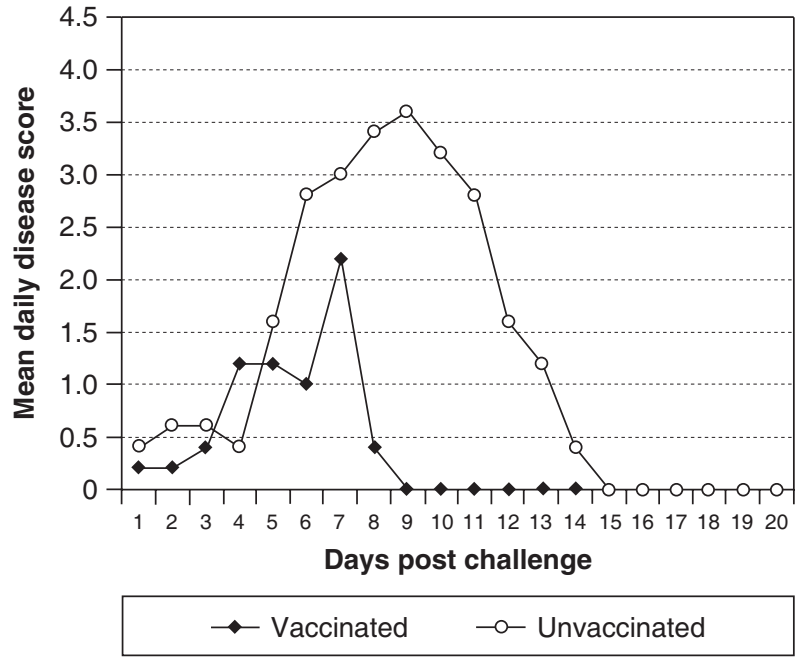

FIG. 3 Disease profiles obtained when vaccinated and unvaccinated chickens were challenged with serovar C-2 of H. paragallinarum

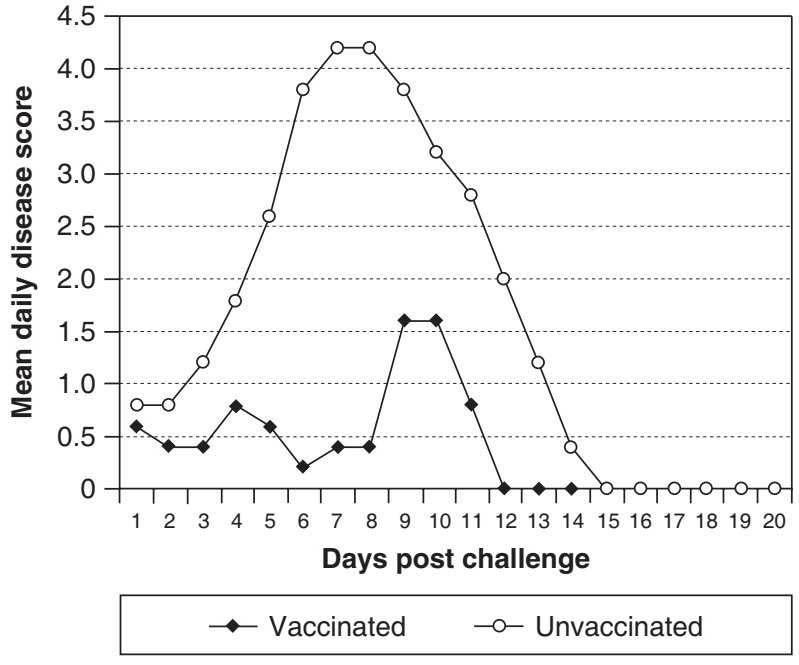

FIG. 4 Disease profiles obtained when vaccinated and unvaccinated chickens were challenged with serovar C-3 of H. paragallinarum

TABLE 1 Mean disease scores, maximum daily disease score, duration of clinical signs and percentage protection obtained in vaccinated and unvaccinated chickens challenged with the different serovars of $H$. paragallinarum

\begin{tabular}{|l|l|l|l|l|l|l|}
\hline Isolate & Vaccinated & Mean $^{*}$ & Max ** & Duration* in days & \% protection & Significance \\
\hline A-1 & No & 1.13 & 3.4 & 13 & & \\
A-1 & Yes & 0.17 & 1.0 & 6 & 85.00 & $P=0.00016$ \\
B-1 & No & 0.46 & 2.4 & 8 & 67.50 & \\
B-1 & Yes & 0.13 & 0.8 & 6 & & \\
C-2 & No & 1.28 & 3.6 & 14 & 7 & \\
C-2 & Yes & 0.34 & 2.2 & 73.40 & $P=0.00349$ \\
C-3 & No & 1.85 & 4.2 & 15 & 75.70 & \\
C-3 & Yes & 0.45 & 1.6 & 11 & & \\
\hline
\end{tabular}

* = Mean disease score

** = Maximum daily disease score

\# = Duration of clinical signs

The levels of protection provided by the experimental vaccine ranged from $67.5 \%$ in the vaccinated chickens challenged with serovar B-1 to $85 \%$ in the birds challenged with serovar A-1 (Table 1). The levels of protection obtained when the birds were challenged with the serogroup $\mathrm{C}$ isolates were found to be $73.4 \%$ for serovar $\mathrm{C}-2$ and $75.7 \%$ for serovar $\mathrm{C}-3$. These levels of protection are similar to those obtained using the challenge model developed by Bragg (2002b) when using commercially available IC vaccines in South Africa (data not shown). Rimler, Davis \& Page (1977) also found protection levels between $80 \%$ and $100 \%$ when chickens vaccinated with serogroup $A$ isolates were challenged with serogroup $A$ isolates, thus demonstrating that the protection levels obtained with this experimental vaccine are within the acceptable protection range for inactivated bacterial vaccines.
When considering the clinical signs manifested by vaccinated chickens challenged with the different serovars of $H$. paragallinarum, it is noted that substantial clinical signs were still recorded. When they were challenged with serovar A-1, the mean disease score obtained over the 20-day period was only 0.17 with a maximum daily disease score of 1 , as compared to a mean disease score of 1.13 with a maximum daily disease score of 3.4 obtained in unvaccinated chickens. This translates into an $85 \%$ level of protection. However, during the peak reaction following challenge, a maximum mean daily disease score of 1 was found in the vaccinated group of chickens. There were thus clinical signs of IC in these chickens, in spite of the fact that $85 \%$ protection was obtained with the vaccine.

It has been demonstrated in this experiment and previously by Bragg (2002b) that the serotype B 


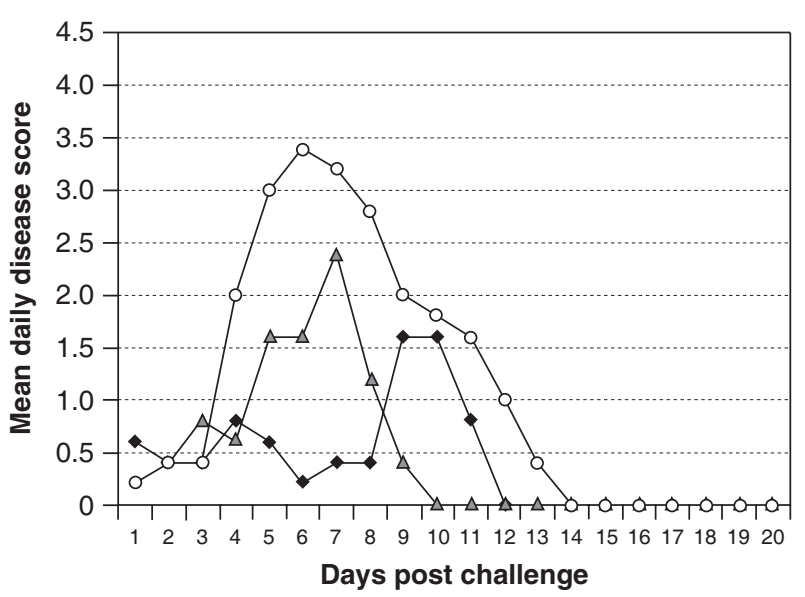

$$
\begin{aligned}
& \rightarrow-\text { Vaccinated C-3 } \\
& \multimap-\text { Unvaccinated A-1 } \rightarrow-\text { Unvaccinated B-1 }
\end{aligned}
$$

FIG. 5 Disease profiles obtained when vaccinated chickens were challenged with serovar $\mathrm{C}-3$ and unvaccinated chickens were challallenged with serovar A-1 or B-1 of H. paragallinarum

isolate in South Africa is of low virulence. When vaccinated chickens were challenged with serovar B-1, clinical signs were still apparent despite a $67.5 \%$ level of protection that was provided by the vaccine.

It has also been established in this experiment and by (Bragg 2004b) that the serogroup C isolates are of high virulence. The clinical signs of IC that developed in vaccinated chickens challenged with serovar C-3, where there was $75.7 \%$ protection, were substantial. A mean disease score of 0.45 was found with the maximum mean daily disease score of 3.6 (Table 1) in the vaccinated chickens. The overall disease profiles obtained in this challenge experiment were similar to those obtained when unvaccinated chickens were challenged with serovar B-1 or serovar A-1 (Fig. 5). If the clinical signs that developed in vaccinated birds challenged with the $\mathrm{C}-3$ serovar are merely observed, but are not compared to those obtained in the unvaccinated birds challenged with the same isolate, it could be postulated that the vaccine did not provide protection. This hypothesis would be even further strengthened if the clinical signs in the vaccinated chickens challenged with C-3 are compared to those obtained in unvaccinated birds challenged with serovar A-1 or B-1, in which the clinical signs were of similar intensity.

Evaluations of the efficacy of an IC vaccine based on challenge models other than the one used in this experiment might lead to confusing results. Jacobs \& Van der Werf (2000) state that the vaccine used in their experiment provided protection against the different serovars of NAD-independent $H$. paragallinarum found in South Africa. Bragg (2004a), on the other hand, suggested that the NAD-independent strains are capable of evading the immune system. These conflicting results could be a result of the low virulence of the NAD-independent isolates. Bragg (2002c) found that the naturally occurring NAD-independent isolates are of low virulence. Taole et al. (2002) were able to convert virulent NAD-dependent serovar C-3 isolates to NAD-independent isolates using a plasmid and they demonstrated that the conversion of the virulent NAD-dependent isolate to NAD independence significantly reduced the virulence of the isolate. If a vaccine challenge model is based purely on the observation of clinical signs and does not score the clinical signs, it would seem that the vaccine used provides good protection, as suggested by Jacobs \& Van der Werf (2000) when, in fact, the isolate used for challenge is merely of very low virulence, as demonstrated by Bragg (2002c) and Taole et al. (2002).

\section{CONCLUSIONS}

Without making use of the challenge model developed by Bragg (2000b) in which the clinical signs are scored and the mean daily disease score for the group of chickens is plotted graphically, a misconception of the levels of protection afforded by a particular vaccine against a specific challenge can be made. The early work on the IC problem in South Africa was based on the assumption that the vaccines used at the time were not providing protection against the serovar C-3 isolate because of the number of serious "vaccine breaks" which were investigated and from which serovar C-3 isolates were made. With the development of this challenge model, it was established that the virulence of the different serovars has a profound impact on the perceived vaccine efficacy. It has been demonstrated in this experiment that the vaccine used provided high levels of protection. However, the virulence of the serogroup $C$ isolates induced substantial clinical signs in the vaccinated chickens. These clinical signs were due to the virulence of the isolates and not to vaccine failure as was previously proposed.

The findings in this experiment suggest that it will not be possible to completely control the IC problem in South Africa through the use of inactivated vaccines alone. Bragg (2004b) has suggested a continual disinfection programme that can be used in conjunction with a vaccination programme to reduce the impact of IC to the poultry producer. Another 
possible approach in the control IC is through the use of live vaccines.

\section{ACKNOWLEDGEMENTS}

I am grateful to Dr Freek Potgieter of the Animal House of the University of the Free State for his assistance with the housing of the birds. I am also grateful to the National Research Foundation for funding aspects of this work.

\section{REFERENCES}

BLACKALL, P.J., EAVES, L.E. \& ROGERS, D.G. 1990a. Proposal of a new serovar and altered nomenclature for Haemophilus paragallinarum in the Kume hemaglutinin scheme. Journal of Clinical Microbiology, 28:1185-1187.

BLACKALL, P.J., EAVERS, E.L. \& AUS, G. 1990b. Serotyping of Haemophilus paragallinarum by the Page scheme: comparison of the use of agglutination and hemagglutination-inhibition tests. Avian Diseases, 34:643-645.

BRAGG, R.R., COETZEE, L. \& VERSCHOOR, J.A. 1993. Plasmid encoded NAD independence in some South African isolates of Haemophilus paragallinarum. Onderstepoort Journal of Veterinary Research, 60:147-152.

BRAGG, R.R., COETZEE, L. \& VERSCHOOR, J.A. 1996. Changes in the incidence of the different serovars of Haemophilus paragallinarum in South Africa: A possible explanation for vaccination failures. Onderstepoort Journal of Veterinary Research, 63:217-226.

BRAGG, R.R., GREYLING, J.M. \& VERSCHOOR, J.A. 1997. Isolation and identification of NAD-independent bacteria from chickens with symptoms of infectious coryza. Avian Pathology, 26:595-606.

BRAGG, R.R. 2002a. Isolation of serovar C-3 Haemophilus paragallinarum from Zimbabwe: A further indication of the need for local vaccines against infectious coryza. Onderstepoort Journal of Veterinary Research, 69:129-132.

BRAGG, R.R. 2002b. Virulence of South African isolates of Haemophilus paragallinarum. Part 1: NAD-dependent field isolates. Onderstepoort Journal of Veterinary Research, 69: 163-169.

BRAGG, R.R. 2002c. Virulence of South African isolates of Haemophilus paragallinarum. Part 2: Naturally occurring
NAD-independent field isolates. Onderstepoort Journal of Veterinary Research, 69:171-175.

BRAGG, R.R. 2004a. Evidence of evasion of protective immunity by NAD-independent isolates of Haemophilus paragallinarum in poultry. Onderstepoort Journal of Veterinary Research, 71:53-58.

BRAGG, R.R. 2004b. Limitation of the spread and impact of infectious coryza through the use of a continuous disinfection programme. Onderstepoort Journal of Veterinary Research, 71:1-8.

BUYS, S.B. 1982. Die bereiding van ' $n$ bakterien teen Haemophilus paragallinarum besmetting, geskik vir Suid-Afrikaanse omstandighede. M.Med.Vet (Bact.) thesis. Faculty of Veterinary Science, University of Pretoria.

HORNER, R.F., BISHOP, G.C. \& HAW, C. 1992. An upper respiratory disease of commercial chickens resembling infectious coryza, but caused by a $\mathrm{V}$ factor independent bacterium. Avian Pathology, 21:421-427

HORNER, R.F., BISHOP, G.C., JARVIS, C.J. \& COETZEE, T.H.T. 1995. NAD (V-factor)-independent and typical Haemophilus paragallinarum infecting commercial chickens: a five year field study. Avian Pathology, 24:453-463.

JACOBS, A.A. \& VAN DER WERF, J. 2000. Efficacy of a commercially available coryza vaccine against challenge with recent South African NAD-independent isolates of Haemophilus paragallinarum in chickens. Journal of the South African Veterinary Association, 71:109-110.

KUME, K., SAWATA, A., NAKASE, Y. \& MATSUMOTO, M. 1983. Serological classification of Haemophilus paragallinarum with a hemagglutinin scheme. Journal of Clinical Microbiology, 17:985-964.

MOUAHID, M., BISGAARD, M., MORLEY, A.J., MUTTERS, R. \& MANNHEIM, W. 1992. Occurrence of V-factor (NAD) independent strains of Haemophilus paragallinarum. Veterinary Microbiology, 31:363-368.

RIMLER, R.B., DAVIS, R.B., PAGE, R.K. 1977. Infectious coryza: Cross-protection studies, using seven strains of Haemophilus paragallinarum. American Journal of Veterinary Research, 38:1587-1598.

TAOLE, M., ALBERTYN, J., VAN HEERDEN, E. \& BRAGG, R.R. 2002. Virulence of South African isolates of Haemophilus paragallinarum. Part 3: experimentally produced NADindependent isolate. Onderstepoort Journal of Veterinary Research, 69:189-196.

YAMAGUCHI, T., BLACKALL, P.J., TAKIGAMI, S., IRITANI, Y. \& HAYASHI, Y. 1990. Pathogenicity and serovar-specific hemagglutinating antigens of Haemophilus paragallinarum serovar B strains. Avian Diseases, 34:964-968. 